CAHIERS DE

NARRATOLOGIE

\section{Cahiers de Narratologie}

Analyse et théorie narratives

37 | 2020

Approches transmédiales du récit dans les fictions contemporaines

\title{
Rhétorique narrative du machinima : polyphonies et saillances d'une narration sous contrainte
}

\section{Fanny Barnabé}

\section{(2) OpenEdition \\ Journals}

Édition électronique

URL : http://journals.openedition.org/narratologie/10441

DOI : $10.4000 /$ narratologie. 10441

ISSN : 1765-307X

Éditeur

LIRCES

Référence électronique

Fanny Barnabé, «Rhétorique narrative du machinima : polyphonies et saillances d'une narration sous contrainte ", Cahiers de Narratologie [En ligne], 37 | 2020, mis en ligne le 04 septembre 2020, consulté le 07 septembre 2020. URL : http://journals.openedition.org/narratologie/10441 ; DOI : https://doi.org/ 10.4000/narratologie.10441

Ce document a été généré automatiquement le 7 septembre 2020

Article L.111-1 du Code de la propriété intellectuelle. 


\title{
Rhétorique narrative du machinima: polyphonies et saillances d'une narration sous contrainte
}

\author{
Fanny Barnabé
}

\section{Introduction}

1 Au sens strict, le machinima est une vidéo (ou une série de vidéos) «composé[e] de captures vidéo de sessions de jeu, ayant fait ou non l'objet d'une postproduction (montage vidéo, insertion d'une bande son, composition de musique, générique, etc.) » (Georges et Auray 2012a: 4). Les machinéastes (ou machinimakers) utilisent donc les moteurs des jeux vidéo afin de produire des films d'animation. Concrètement, ces créateurs se servent des avatars comme marionnettes et des environnements comme décors pour jouer des saynètes qui seront enregistrées et, éventuellement, montées, doublées ou retouchées. Le machinima se distingue donc d'autres formes de vidéos telles que le speedrun ou le let's play en ce qu'il ne représente pas un playthrough, mais met les composantes du jeu au service d'une mise en scène pouvant relever de plusieurs genres (fiction, clip musical, sketch, documentaire, talk-show, etc.) ayant pour spécificité de ne pas être originaire du domaine vidéoludique. Le machinima, en somme, ne se contente pas de détourner les jeux pour produire des vidéos: il remédiatise (Rajewsky 2005) aussi des genres canoniques en les adaptant à un mode de représentation vidéoludique.

Cet objet a déjà bénéficié de l'éclairage d'un certain nombre d'études, où dominent les perspectives historiques (retraçant l'évolution des vidéos ou les envisageant comme des outils de conservation et de documentation des expériences vidéoludiques; voir Lowood 2006, 2008 et 2011; Kelland 2011; Nitsche 2005 et 2007), techniques (qui s'interrogent sur les formats des œuvres ou se présentent comme des manuels d'aide à la création; voir Kirschner 2011; Manovich 2011; Mazalek 2011; Marino 2004) et esthétiques. Parmi ces derniers travaux, on retrouve une attention particulière portée à l'hybridité formelle et à l'intermédialité du genre (Nitsche 2005, 2007 et 2011; Krapp 
2011), à ses conventions stylistiques (Pigott 2011 ; Bardzell 2011), à son rapport au jeu (Pinchbeck et Gras 2011) ou encore à sa capacité à "réconcilier jeu et narration " (Schneider 2008). D'autres travaux délaissent les analyses formelles et s'intéressent plutôt au machinima en tant que pratique ou phénomène communautaire, en le réinscrivant notamment dans le contexte de la culture participative : c'est le cas de Schott (2011), Cameron et Carroll (2009), Salen (2011) et de Picard (2007). On trouve, dans le même ordre d'idées, des approches proprement ethnographiques (Schott et Yeatman 2011) et sociologiques (Georges et Auray 2012a et 2012b, retracent ainsi des trajectoires de machinéastes et s'interrogent sur leurs stratégies de consécration et de positionnement dans le champ).

3 S'inscrivant dans la continuité des études formalistes du genre, le présent travail vise à interroger les spécificités narratives et discursives du machinima au moyen de l'analyse de certaines de ses figures rhétoriques prototypiques. Les figures sont ici entendues en suivant Bonhomme (2014:31) - comme des passages où l'énoncé produit une forme de résistance, où des marqueurs (morphologiques, syntaxiques ou énonciatifs) font émerger une saillance. En d'autres termes, une figure rhétorique a pour particularité de :

Présenter un relief qui peut être proéminent ou en creux (pensons à l'ellipse) et qui l'individualise dans le continuum des énoncés, ce dernier étant relatif à chaque situation de communication. [...] De la sorte, produire une figure, c'est engendrer une structure saillante (et non plus anormale ou déviante, comme pour les théories de l'écart) qui se manifeste par un épaississement de la substance langagière dans certaines séquences discursives (Bonhomme $2014: 40$ ).

4 En raison de leur intense hybridité formelle (ces vidéos empruntent des codes au cinéma, au théâtre, notamment de marionnettes, à la danse, à la télévision et au clip musical, mais sont aussi profondément déterminées par leur substrat vidéoludique) et de leur principe même de recyclage d'univers vidéoludiques préexistants, nous verrons que les machinimas multiplient les figures rhétoriques produisant des effets d'écart, de disjonction ou de polyphonie. Ces vidéos sont, en cela, représentatives d'une esthétique du détournement ${ }^{1}$. Or, en inscrivant explicitement les machinimas dans le domaine des productions « de seconde main " (Compagnon 1979) ou " au second degré " ${ }^{2}$, ces figures rhétoriques s'articulent de manière complexe avec la construction d'un récit supposément linéaire, cohérent et fini. Certaines d'entre elles, comme les mises en abyme et les métalepses, "conteste[nt] la marche du récit " (Fevry $2007: 23$ ) par leur caractère réflexif ; d'autres mettent au jour la nature de "narration sous contrainte " propre au machinima. Enfin, en ce qu'elles sont traversées de références et d'intertextes, de couches de fiction superposées ou entremêlées, ces vidéos construisent des récits se distinguant, eux aussi, par leur hétérogénéité.

5 L'objectif de cet article sera donc de déterminer comment la rhétorique du machinimamalgré ses effets de polyphonie - peut être mise au service de la narration, mais aussi de qualifier le type d'univers fictionnel que ces vidéos participent à construire. Dans ce but, nous procéderons à l'analyse parallèle de deux séries de machinimas ayant pour point commun de remédiatiser la série animée Pokémon au sein de deux jeux vidéo différents. Ces séries de vidéos constituent en effet des illustrations particulièrement évocatrices de l'intime intrication de strates fictionnelles, de conventions médiatiques et de références communautaires qui est au cœur du langage du machinima. 


\section{Une superposition de remédiatisations : les machinimas Halomon et Garrymon}

6 La pratique consistant à retraduire une diégèse (qu'elle soit ludique, filmique ${ }^{3}$, littéraire ${ }^{4}$, etc.) dans le cadre (ludique et fictionnel) d'un jeu vidéo est extrêmement courante et traverse tous les genres de machinimas. Les deux mini-séries Halomon ${ }^{5}$ et Garrymon $^{6}$ (composées de deux vidéos chacune) s'inscrivent dans ce format, puisqu'elles reproduisent méthodiquement les deux premiers épisodes du dessin animé Pokémon (lui-même adapté des titres vidéoludiques) au sein des jeux vidéo Halo: Reach (Bungie Studios, 2010) et Garry's Mod (Facepunch Studios, 2006 pour la version commerciale).

7 Le dessin animé Pokémon - dont la diffusion a commencé au Japon dès 1997 - narre les aventures d'un jeune garçon nommé Sacha, qui parcourt le monde dans le but de devenir «Maître Pokémon ». Les deux premiers épisodes montrent le départ de son périple depuis son village natal de Bourg Palette, où le Professeur Chen lui confie son premier Pokémon : un Pikachu caractériel qui refuse de rentrer dans sa Pokéball. Une fois sur la route, le duo se fait attaquer par un groupe de Pokémon sauvages et Sacha doit conduire d'urgence son Pikachu au Centre Pokémon le plus proche pour le soigner. En chemin, il fait la connaissance d'Ondine (une jeune dresseuse dont il a détruit le vélo et qui décidera de le suivre pour s'assurer d'être remboursée), d'une policière, d'une infirmière et, enfin, de trois membres de la Team Rocket, une organisation criminelle spécialisée dans le vol et le trafic de Pokémon. Ces derniers s'introduisent dans le Centre Pokémon à la recherche de créatures rares à subtiliser, mais Sacha et ses nouveaux compagnons parviennent à les arrêter et le deuxième épisode s'achève sur la reprise du voyage initiatique.

8 Les machinimas Halomon (BangBang TV) et Garrymon suivent assez fidèlement la trame narrative de ces deux épisodes (bien que certaines scènes secondaires soient abrégées), mais traduisent les événements (par le biais de métaphores et de resémantisations qui seront détaillées plus bas) dans deux autres univers : celui du FPS ${ }^{7}$ futuriste Halo: Reach, pour Halomon, et celui du jeu « bac à sable » Garry's Mod, dans le cas de Garrymon.

Dans Halomon, les vidéos racontent l'histoire du jeune garçon "Crachat », qui reçoit son premier «Halomon » (un «Pikafion») de la part du Professeur "À-la-chaîne » ${ }^{8}$ et combat les malfaiteurs de la "Team Croquette». Les péripéties du dessin animé parfois reproduites plan par plan - sont représentées avec les moyens offerts par le jeu de tir Halo: les personnages apparaissent en armures et ne sont reconnaissables que grâce à leurs couleurs, leurs accessoires et leurs voix ; les Pokéballs sont représentées par des grenades, le vélo d'Ondine par un Warthog (un véhicule de combat) et les Pokémon eux-mêmes (ou plutôt les "Halomon») sont incarnés par des avatars de joueurs (des humains ou des extraterrestres, selon les cas) (fig. 1). 


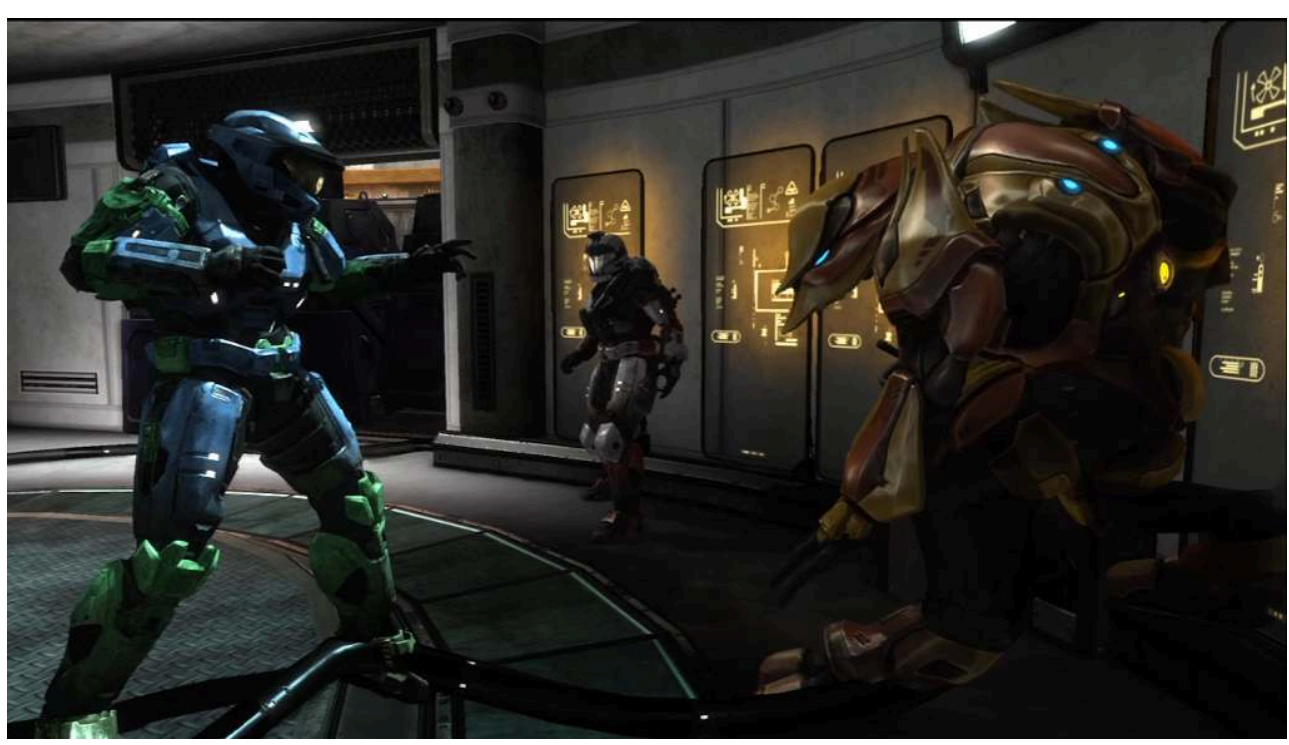

Fig. 1 (Tous Droits Réservés) - Le Professeur À-la-chaîne confie à Crachat (à gauche) son premier Halomon (à droite).

Notons que les noms et caractéristiques de ces créatures renvoient bien souvent, non à l'univers de Pokémon, mais aux pratiques de jeu multijoueurs de Halo. À 09:109 du premier épisode, par exemple, le protagoniste rencontre un Halomon nommé «Newbie» (terme désignant un joueur débutant), qui est décrit comme particulièrement docile et facile à capturer ; plus loin (à 11:34), il croisera un «Groslâche ", c'est-à-dire un Halomon qui se déplace toujours en groupe et dont la capacité spéciale (le Team Shot) «consiste à attaquer ses adversaires en surnombre». Dans l'introduction du même épisode (à 01:52), il est encore fait mention d'un « Halomon de type $\mathrm{PGM} »$ : l'acronyme provient de l'expression pro gamer et désigne un joueur professionnel ou extrêmement talentueux.

11 Des références du même type sont également présentes dans le générique du machinima, qui parodie celui de la série en l'imitant très fidèlement d'un point de vue visuel (fig. 2), mais en réécrivant ses paroles pour faire écho au mode multijoueurs de Halo. La phrase originale « un jour je serai le meilleur dresseur, je me battrai sans répit ; je ferai tout pour être vainqueur et gagner les défis » devient ainsi : « un jour je serai le meilleur tueur, j'obtiendrai tous les défis; je ferai tout pour être vainqueur et gagner des crédits». Le terme "défi» devient ici un calembour en ce qu'il active simultanément deux significations différentes (et condense en son sein le mélange de substrats à l'œuvre dans le machinima) : il renvoie au générique original, mais aussi à la désignation des quêtes régulières (journalières, hebdomadaires ou mensuelles) proposées par les jeux Halo. De même, plus loin, «notre amitié triomphera » devient " notre connexion triomphera », etc. 

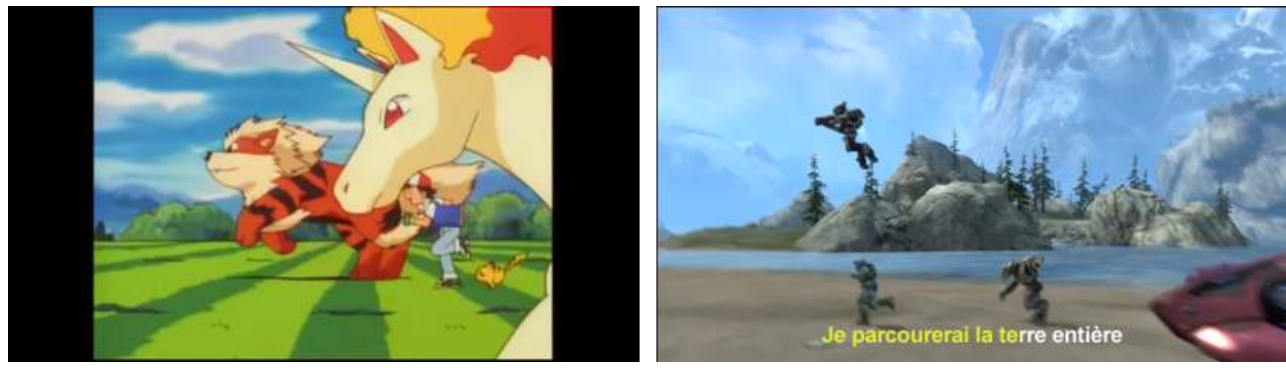

Fig. 2 (Tous Droits Réservés) - Le générique original (à gauche) et sa reproduction en machinima dans Halo : Reach

Les vidéos mêlent donc trois univers de sens a priori peu compatibles: la fiction Pokémon telle qu'elle est représentée dans la série télévisée, la fiction de Halo: Reach (avec ses composantes visuelles et narratives: la présence d'aliens, de soldats en armures, d'armes et de véhicules de combat) et la pratique du jeu (dont le métadiscours n'est pas censé avoir de signification au sein de la diégèse).

L'hétérogénéité est aussi la caractéristique la plus flagrante des machinimas Garrymon (SpawnThemAll), qui sont basés sur un principe similaire, mais produits indépendamment par un autre studio de machinéastes (anglophones, cette fois). Ceuxci utilisent comme support Garry's Mod créé par Garry Newman, un mod (modification d'un système de jeu) de Half-Life 2 (Valve, 2004) se situant à cheval entre le jeu « bac à sable » et le logiciel d'aide à la création. Garry's Mod a, en effet, l'apparence d'un jeu, dans le sens où il permet à son utilisateur d'arpenter un monde en trois dimensions et d'utiliser diverses armes ou divers outils. Ce monde est initialement vide et ne contient pas vraiment d'objectifs prédéterminés. Cependant, le joueur peut faire apparaître des objets ou personnages ${ }^{10}$, les manipuler, les associer, et les combiner à sa guise afin de concevoir lui-même des machines, des niveaux ou des décors. Garry's Mod se présente donc comme une boîte à outils permettant de jouer avec les possibilités du moteur physique Source : il est, de ce fait, un instrument très populaire pour la création d'autres mods et de machinimas.

4 En outre, Garry's mod dispose d'une fonctionnalité assez unique en son genre et particulièrement utile pour la réalisation de mises en scène: le ragdoll posing ${ }^{11}$. Les outils offerts par ce mod permettent ainsi de positionner et de manipuler à loisir les corps des personnages que l'on peut faire apparaître : il est notamment possible de les déplacer à volonté, de leur faire prendre n'importe quelle posture et de modifier très précisément leur expression faciale, la direction de leur regard et jusqu'à la position de chacun de leurs doigts. Les joueurs ont donc naturellement exploité ces fonctionnalités pour produire des captures d'écran ${ }^{12}$, des bandes dessinées ${ }^{13}$ et des machinimas (souvent réalisés en stop motion). L'un des attraits humoristiques de ce type de productions réside dans le caractère saugrenu des postures des personnages et dans l'aspect désarticulé de leurs mouvements ${ }^{14}$.

Les deux épisodes de Garrymon adaptent ainsi les péripéties de la série Pokémon dans l'univers visuel très particulier de Garry's Mod et, plus spécifiquement, dans le cadre fictionnel d'Half-Life 2, auquel le machinima emprunte la plupart de ses personnages, objets et ressources graphiques. Ce dernier jeu se déroule dans un univers dystopique où les humains luttent pour survivre à l'oppression d'une société extraterrestre: la noirceur de la fiction et la monstruosité du bestiaire sont donc déjà dissonantes par rapport à l'esthétique des dessins animés Pokémon. 

référence à un autre machinima humoristique réalisé avec Garry's Mod et devenu un classique, Half-Life: Full Life Consequences ${ }^{15}$, dont le protagoniste est un certain John Freeman, prétendu frère de Gordon Freeman, le personnage central des deux premiers jeux Half-Life. Ce seul clin d'œil témoigne déjà de la densité des jeux intertextuels qui forment la structure et l'univers de sens des machinimas, non seulement car il souligne le crossover ${ }^{16}$ présupposé par le réemploi d'éléments graphiques préexistants, mais aussi car il renvoie à un autre détournement que le succès a institué en nouvelle norme du genre.

L'intrigue débute en outre dans le village de "gm_pallet", dont le nom renvoie simultanément au Bourg Palette de l'univers Pokémon et au formatage du nom des niveaux de jeu dans le logiciel Garry's Mod. John Freeman s'y rend chez le Professeur Kleiner (l'un des personnages principaux de Half-Life 2, qui joue ici le rôle du Professeur Chen) afin d'obtenir son premier Garrymon : un «crabe de tête » (headcrab) surnommé Lamarr (fig. 3). Ces headcrabs sont des créatures emblématiques des jeux Half-Life (il s'agit de parasites extraterrestres qui s'accrochent à la tête des humains pour les transformer en zombies) et Lamarr est l'une d'entre elles, que le Docteur Kleiner tente d'apprivoiser dans le récit de Half-Life 2.

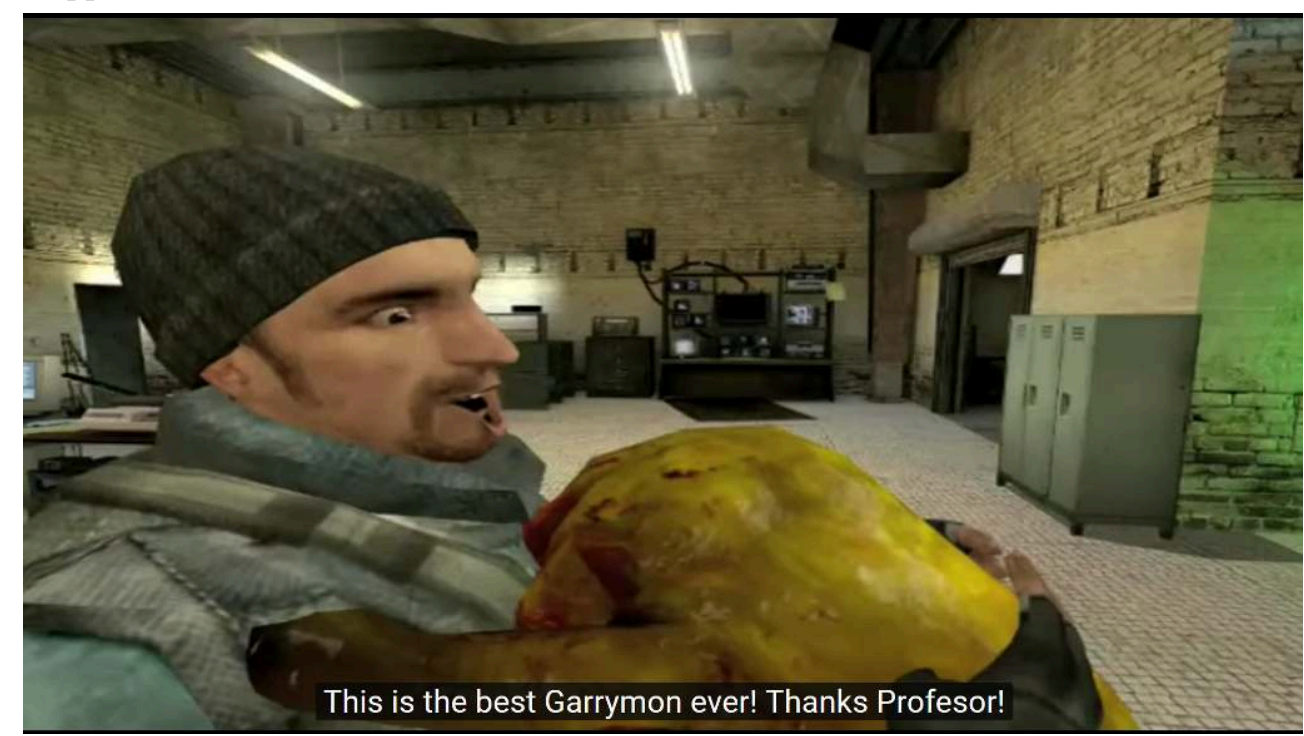

Fig. 3 (Tous Droits Réservés) - John Freeman reçoit son premier Garrymon : un headcrab nommé Lamarr

Par la suite, le machinima Garrymon continue de suivre la trame de la série télévisée, tout en citant continuellement Half-Life et Garry's Mod (mais en tant que logiciel et non plus en tant que support d'une pratique du jeu, comme c'était le cas dans Halomon). Ainsi, la créature que le protagoniste tente de capturer dans le premier épisode se nomme, cette fois "CityScanner» (en référence aux drones de surveillance de HalfLife 2) et est décrite comme "un Garrymon de type Noclip » (06:28), ce dernier terme renvoyant à une commande que le joueur peut entrer dans la console de Garry's Mod afin de se déplacer librement, en volant dans les airs et en passant à travers les obstacles, sols et murs.

De même, dans le deuxième épisode, John Freeman affronte, non plus la Team Rocket, mais la "Team Thruster» ("propulseur»), qui est composée de personnages de HalfLife 2 (Wallace Breen et Judith Mossman) et dont le nom rappelle un outil de 
construction utilisable dans Garry's Mod (le thruster permet d'ajouter un effet de propulsion aux objets). Il ne s'agit là que de quelques exemples noyés dans le flot des citations intertextuelles qui accompagnent tout le déroulement de la narration.

Les vidéos de Halomon et de Garrymon poursuivent donc un objectif narratif similaire : raconter l'histoire des premiers épisodes de Pokémon à l'aide d'outils de représentation particulièrement peu propices à cette imitation et - dans la foulée - utiliser ce "pastiche médiatique» (Georges et Auray 2012a : 25) comme prétexte pour raconter une expérience collective des jeux vidéo mobilisés. Dans les pages suivantes, nous tâcherons de lister différentes figures rhétoriques faisant "saillance" (Bonhomme 2014: 31) au sein de ces deux séries de vidéos et de montrer leur impact sur la construction du récit et du monde fictionnel. Ces procédés seront réunis en deux groupes: les figures opérant des ruptures du cadre narratif (fragmentations, paradoxes, métalepses, et mises en abyme) et celles manifestant l'indexicalité du machinima ${ }^{17}$, sa dimension de «narration sous contrainte» (resémantisations, métaphores et caricatures).

\section{Ruptures du cadre narratif : la narration comme « exercice des possibles »}

21 Le machinima peut être envisagé comme un moyen d'enregistrer et de fixer une expérience de jeu: Pinchbeck et Gras le définissent ainsi comme «le processus de production d'un artéfact linéaire à partir d'un système non linéaire, de réduction du champ des affordances internes à ce système en un flux individualisé ${ }^{18}$ (Pinchbeck et Gras 2011 : 144). Schott et Yeatman, quant à eux, soulignent que « la multiplicité des choix potentiellement générés par l'interaction au sein d'un environnement virtuel devient contenue comme une trajectoire singulière et répétable de choix réels faits par le producteur du machinima ${ }^{19}$ (Schott et Yeatman $\left.2011: 116\right)$.

Si, dans cette perspective, la production d'une vidéo apparaît comme une fermeture des possibles de l'œuvre originale, d'un point de vue narratif, les machinimas procèdent régulièrement à une ouverture des significations du jeu par le biais de figures " décloisonnantes ", c'est-à-dire ayant pour effet de rompre le cadre de la diégèse. Dans ces vidéos, la narration peut se concevoir comme un véritable " exercice des possibles » (exercice définitoire, selon $\mathrm{Genvo}^{20}$, de l'activité ludique), une interprétation libérée des contraintes imposées par les codes narratifs préexistants, les impératifs commerciaux et même par la logique. Aux machinimas peut s'appliquer, en d'autres termes, cette remarque formulée par Derecho au sujet des fanfictions :

Dans la fanfiction, il y a une reconnaissance que chaque texte contient des potentialités infinies, dont n'importe laquelle pourrait être actualisée par n'importe quel écrivain intéressé de faire le travail : les auteurs de fanfictions [...] explorent des situations que les créateurs du texte source ne peuvent simplement pas [explorer], à cause du besoin de continuité et de cohérence chronologique dans l'univers $d u$ texte source (et l'absence d'une telle exigence dans les productions de fans) ${ }^{21}$ (Derecho 2006 : 76).

Dans Halomon et Garrymon, ce caractère libéré (voire ludique) de narration transparaît par le biais de quatre types de procédés, détaillés ci-dessous. 


\section{Fragmentation et paradoxes}

24 contradiction interne, mais parce qu'ils ne s'intègrent pas dans l'univers de sens construit par le co-texte. Lorsque la mère de John Freeman (dans Garrymon, épisode 1) oblige ce dernier à regarder une émission éducative pour se préparer à son voyage (programme qui a l'air de le passionner), la séquence crée de l'attente pour un certain contenu. Or l'émission en question consiste uniquement en un plan sur le Professeur Kleiner en train de contorsionner à toute vitesse ses bras dans des angles peu naturels tout en émettant des bruits incompréhensibles (d'où ressortent certaines phrases telles que "attrapez-les tous!»; 01:59). Dans le deuxième épisode, quand le médecin emmène Lamarr dans la salle d'opération pour le soigner (08:57), l'urgence de la situation (pourtant construite par toute la narration qui précède) est immédiatement démentie par les paroles qu'on lui entend formuler une fois la porte fermée: «Everyone! Lunchtime! I made schnitzels!»-paroles principalement dictées par le fait que le modèle 3D de personnage utilisé pour jouer ce rôle (provenant du jeu de Team Fortress 2) est d'origine allemande.

27 L'absurdité peut par endroits se faire plus prégnante encore par le biais d'une saturation: c'est le cas quand la Team Croquette de Halomon parodie la devise de la Team Rocket (une tirade que ceux-ci répètent à chacune de leurs apparitions) en remplaçant tous ses termes pour n'en conserver que les rimes, sans se préoccuper $d u$ sens. Le discours forme ainsi une série de «calembours intertextuels », puisque les jeux de mots ne sont compréhensibles qu'en comparant les deux textes (épisode 2: 13:08-13:30) : 
Tirade originale de la Team Rocket :

Afin de préserver le monde de la dévastation, Afin de réserver le micro-ondes avec

Afin de rallier tous les peuples à notre nation, Afin de rallier tous les popols à notre nation,

Afin d'écraser l'amour et la vérité,

Afin d'étendre notre pouvoir jusqu'à la voie lactée,

Jessie !

James !

La Team Rocket plus rapide que la lumière,

Rendez-vous tous, ou ce sera la guerre,

Miaouss, oui, la guerre ! congélation,

Sa parodie dans Halomon :

Afin de détruire la moule et la virilité,

Afin d'étendre notre couloir jusqu'à la voie d'à côté,

Vessie !

Nems !

La Team Croquette, plus acide que la rivière,

Pendez-vous tous, ou ce sera la misère,

Maouss, oui, la misère !

Dans la série originale, la devise est suivie d'une réplique du protagoniste s'interrogeant sur les motivations exactes des criminels: "qu'est-ce qu'il y a à comprendre ? Tout ça n'a aucun sens! ». Dans le machinima, la réécriture de la tirade permet de donner une autre signification à cet échange, puisque le discours est effectivement absurde et hors sujet.

D'autre part, les différentes «sorties » de la cohérence fictionnelle témoignent aussi d'une construction ludique de la narration, d'une ouverture de ses possibles ${ }^{22}$, en raison de leur caractère gratuit: de nombreuses actions des personnages ne sont ni introduites par des causes, ni suivies de conséquences. Dans Garrymon, par exemple, le Professeur Kleiner confie soi-disant à John Freeman une Garryball contenant une créature, puis s'écrie immédiatement après qu'il ne s'agit pas d'un Garrymon, mais bien d'une bombe expérimentale (épisode 1: 04:09): celle-ci explose au visage du protagoniste, puis la conversation continue comme si rien n'était advenu.

Dans le même ordre d'idées, l'agent de police rencontré par John Freeman commence d'emblée par lui tirer dessus (car il le juge suspect) en clamant la réplique du film Platoon (Oliver Stone 1986): "Dance, motherfucker!»; ensuite, désirant contrôler l'identité du héros, il se laisse tomber d'un parapet à la manière d'une poupée de chiffon puis effectue - sans raison apparente - une roulade pour attraper le Gabedex du protagoniste (épisode 2: 03:45-04:23). Un peu plus loin, les deux personnages se rendent en véhicule au Centre Garrymon et écrasent, à leur arrivée, un homme qui faisait la queue au comptoir - action qui n'aura aucune conséquence sur la suite du récit ni même sur les dialogues (07:23).

1 L'enchaînement de ces gestes libérés de leur propre contexte participe à décloisonner la narration des impératifs de causalité, de logique et de cohérence qu'on lui impose traditionnellement. En raison de la succession des paradoxes et contradictions, il est difficile d'identifier, dans Halomon et Garrymon (excepté dans les moments où ils décalquent la série Pokémon), un système de valeurs stable et cohérent qui régirait l'univers.

2 Un objet apparaissant dans le deuxième épisode de Garrymon illustre tout particulièrement cette dynamique narrative basée sur la juxtaposition d'éléments sans nécessité de justification, à savoir : l'« Attrape-Criminel » (Criminal Catcher). Il s'agit du 
véhicule que l'agent de police utilise pour conduire le héros en urgence à l'hôpital et dont il détaille ainsi la composition :

Il a une guitare sur le côté pour le rendre cool, une main super-deluxe conçue spécifiquement pour attraper des criminels, un TomTom ${ }^{23}$ lourdement modifié pour détecter le comportement criminel dans les cinq cents mètres, et un propulseur de quinze mégawatts à l'arrière pour vous faire voler à travers la région Lambda ${ }^{24}$ vraiment vite ${ }^{25}$ (05:07-05:35).

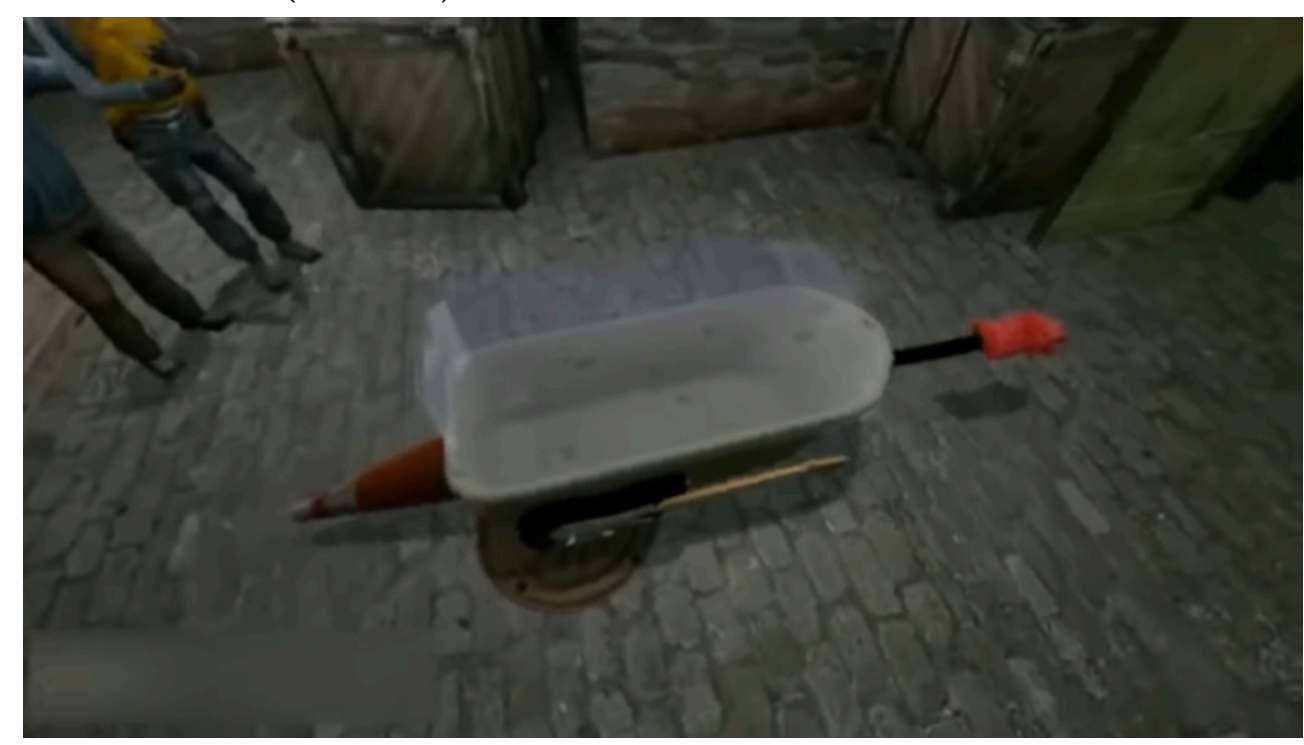

Fig. 4 (Tous Droits Réservés) - Le Criminal Catcher, véhicule des forces de l'ordre dans Garrymon

Dans les faits, le Criminal Catcher se compose principalement d'une baignoire à laquelle sont accolés divers objets (une guitare, un gant en caoutchouc, un cône en guise de " propulseur ", etc. ; voir fig. 4). Ce patchwork insolite dont l'assemblage est dicté plus par la plaisanterie que par les besoins de la mise en scène (peu d'éléments dans l'objet rappellent sa fonction de véhicule) condense non seulement toute la logique de collage propre au machinima, mais rappelle aussi la pratique du jeu Garry's Mod, dont l'un des objectifs suggérés est la construction de machineries à partir d'objets préexistants. Il témoigne ainsi d'une autre caractéristique de cette forme de détournement: le machinima constitue non seulement un outil de documentation sur le jeu tel qu'il est, mais il rend aussi compte du jeu dans ses potentialités créatives.

\section{Métalepses et mises en abyme}

D'autres figures très fréquemment rencontrées dans les machinimas participent à ouvrir les frontières de la fiction représentée : les métalepses et les mises en abyme. Les premières désignent la transgression d'un seuil de la fiction, dans un sens (le monde référentiel apparaît dans la fiction, ce qui forme une métalepse à proprement parler, selon Genette, 2004: 27) comme dans l'autre (la fiction pénètre dans le monde référentiel: une antimétalepse, pour Genette). Les mises en abyme, quant à elles, supposent bien souvent ce type de transgression, puisqu'elles enchâssent plusieurs niveaux de représentation les uns dans les autres de manière réflexive.

Or les «sorties" de la fiction, dans Halomon et Garrymon, ne renvoient pas tant au monde empirique en général qu'à la pratique vidéoludique en particulier. Ainsi, on a déjà vu que le générique de Halomon réécrit les paroles de l'original pour faire 
référence, non à Pokémon ou à l'univers de Halo, mais à l'expérience multijoueurs du jeu (avec des phrases telles que "notre connexion triomphera »). Il en va de même pour le générique de Garrymon, qui mêle des phrases calquées sur celui de Pokémon à des références au jeu Half-life ( je veux être le meilleur, comme Half-Life ne l'a jamais été » $\left.{ }^{26}\right)$, aux cartes ( maps ») ou encore au « hacking ».

Dans Garrymon, encore, la devise de la Team Rocket est réécrite sur le même principe, puisqu'elle ne contient presque plus que des allusions au jeu vidéo (où à l'informatique) n'ayant pas de sens dans la diégèse du machinima :

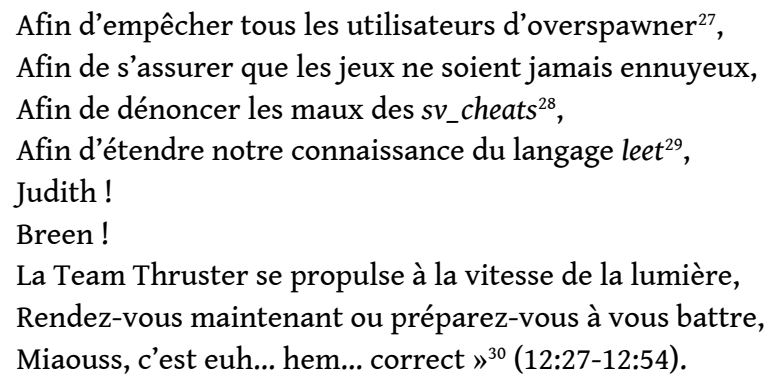

Dans ces métalepses, l'intrusion de références au monde empirique dans la diégèse n'est en rien justifiée ou atténuée : la pratique du jeu vidéo n'est pas resémantisée (pour symboliser une action qui aurait du sens dans l'univers des vidéos), mais mobilisée pour ce qu'elle est. Loin de fondre tous les univers de sens auxquels il renvoie dans un produit homogène et cohérent, le machinima entretient leur dissonance et en rend apparente sa propre artificialité.

Les figures rhétoriques relevées ci-dessus ont donc un statut particulier, car elles ne mettent pas en abyme les œuvres Pokémon, Halo ou Garry's Mod, ni même la pratique du machinima, mais bien l'expérience de jeu. Ce faisant, elles situent la vidéo dans le prolongement du jeu, comme s'inscrivant dans une généalogie ludique. Là où d'autres genres demandent au récepteur d'oublier qu'il est face à une représentation et d'oublier le médium qui la supporte (pour favoriser la cohérence narrative, la création d'une impression référentielle), le machinima ne cesse de révéler ce statut, de rappeler le récepteur à sa position de spectateur et de joueur. De la même façon que les fictions mises en abyme font «de la situation de feintise ludique elle-même un élément thématique " (Schaeffer, 1999: 162), ces vidéos semblent prendre le jeu (et le détournement) comme motif narratif fondateur et comme structure.

\section{Une narration sous contrainte}

Dans le machinima, «l'environnement ludique n'est pas un espace de performance passif; le moteur de jeu peut repousser les actions du performer/joueur d'une manière qui fait obstacle à la production du machinima, mais qui serait en adéquation avec un comportement axé sur le jeu $»^{31}$ (Cameron et Caroll 2009: 6). Autrement dit, dans ce mode de production de vidéos, "le gameplay et les règles deviennent autant de contraintes supplémentaires » (Georges et Auray 2012a : 7) avec lesquelles les joueurscréateurs doivent négocier, «faire avec» (De Certeau 1980:50). Le fait de détourner ces contraintes, de «trouver des outils et des astuces pour contourner l'absence de [...] fonctionnalités sur l'interface de jeu » (Georges et Auray 2012a : 12) est au cœur de la définition du machinima, car, sans cette dimension, il ne serait plus une pratique culturelle spécifique, mais seulement une technique parmi d'autres de l'animation. Le 
machinima tient donc, d'une certaine manière, de la "narration sous contrainte", puisque les machinéastes doivent concevoir leurs mises en scène à l'aide d'un matériau prédéterminé et d'un nombre limité d'animations, de décors et de personnages.

Il découle de ces contraintes que, parfois, "de simples actions peuvent poser des problèmes complexes » (Nitsche $2005: 9$ ). Kelland souligne ainsi que, dans les jeux où la vidéo est enregistrée à travers le point de vue d'un avatar, tous les mouvements de «caméra » et toutes les prises de vues ne sont pas possibles. Il prend également l'exemple plus précis de la série Les Sims (Maxis 2000-2018), où, pour faire en sorte que deux personnages s'embrassent, il est nécessaire de d'abord jouer un certain temps pour mettre ceux-ci de bonne humeur et faire en sorte qu'ils s'apprécient (Kelland 2011:29-30). Dans Halo - où les actions et expressions des avatars sont particulièrement limitées - la simulation d'un simple geste demande bien souvent de mouvoir tout le corps du personnage. Dans Halomon (épisode $1: 12: 57$ ), pour indiquer son chemin au protagoniste et appuyer visuellement sa ligne de dialogue («par ici !»), la dresseuse Ondine doit par exemple se tourner fastidieusement dans la direction mentionnée puis revenir à son point de départ, ce qui inscrit d'emblée la discussion (pourtant banale) dans un registre d'exagération.

41 De la même manière, quand le Halomon du protagoniste est blessé par un tir (épisode 1: 12:18), l'avatar de Halo qui le représente doit, par la suite, alterner constamment entre la position accroupie et la marche normale pour feindre un déplacement pénible à cause de sa blessure. Ces mouvements erratiques sont peu lisibles sans les indices donnés par les dialogues et sans connaissance préalable des événements contés par la série Pokémon. Le minimalisme de ces gestes participe, en quelque sorte, à ludiciser ${ }^{32}$ la réception du machinima, dont l'enjeu devient, dès lors, de réussir à reconnaître, dans les mises en scène parfois peu évocatrices de la vidéo, les actions, objets ou événements du matériau source.

Le fait que ces entraves et limitations ne soient pas contournées (par le choix d'un logiciel plus permissif, par exemple) montre bien leur importance dans la définition et dans l'esthétique du machinima : si les vidéos étaient désolidarisées de ces contraintes, elles perdraient une grande partie de leur signification et de leur spécificité (Georges et Auray 2012a: 9). La dualité, l'exagération, la superposition des sens participent effectivement à la densité des vidéos.

\section{De la resémantisation à la « métaphore »}

43 Pour compenser l'absence de certaines possibilités expressives dans les jeux et leur incapacité à produire des mimétismes fidèles, les machinimas sont contraints de récupérer des gestes parfois proprement vidéoludiques (comme les glitchs ${ }^{33}$ ) et de les resémantiser en les recontextualisant. Dans Halomon, par exemple, l'acte de lancer une grenade - une fois sorti de son contexte ludique et replacé dans celui d'une série de vidéos parodiant Pokémon - permet de représenter le lancer de Pokéball sur un Pokémon (fig. 5). L'outil guerrier et le geste agressif sont ainsi pourvus de significations d'un tout autre registre - les Pokéballs étant plutôt thématisées comme des objets positifs, malgré leur relation avec l'acte de capture. 

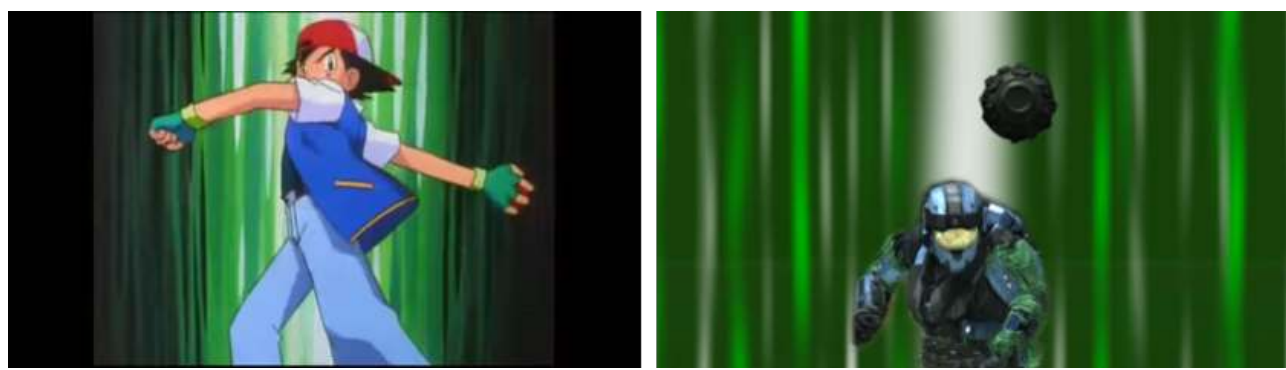

Fig. 5 (Tous Droits Réservés) - L'animation du lancer de grenade dans Halo sert à simuler la séquence récurrente du lancer de Pokéball dans Pokémon

Le machinima «fait avec» (De Certeau 1980: 50), transforme des gestes et objets initialement pourvus d'un sens principalement ludique ou technique en faisant d'eux les composantes signifiantes d'une mise en scène et d'une narration. Autrement dit :

Là où le personnage contrôlé par le joueur peut être pensé algorithmiquement en termes de capacités, de possibilités ou de propriétés affectant le gameplay pendant le jeu ou le superplay [...], dans le machinima, ils sont repensés en tant que parties d'un système de représentation ${ }^{34}$ (Newman 2008 : 145).

Ainsi, le Falcon de Halo: Reach - un hélicoptère de combat utilisable dans les batailles apparaît dans Halomon (épisode 2: 05:07) pour figurer la montgolfière de la Team Rocket (qui, dans le dessin animé, a la forme de la tête du Pokémon chat, Miaouss). Son changement de fonction, dicté par le passage depuis le jeu vers la série de fiction, est rendu évident par l'utilisation qu'en font les personnages : au lieu de s'installer dans le vaisseau, aux tourelles de mitrailleuses, ceux-ci se tiennent simplement debout sur son toit (fig. 6). Un tel positionnement n'aurait que peu de sens dans le cadre du jeu, mais permet, dans la mise en scène, de composer un plan où l'hélicoptère et les deux personnages sont montrés dans leur entièreté.
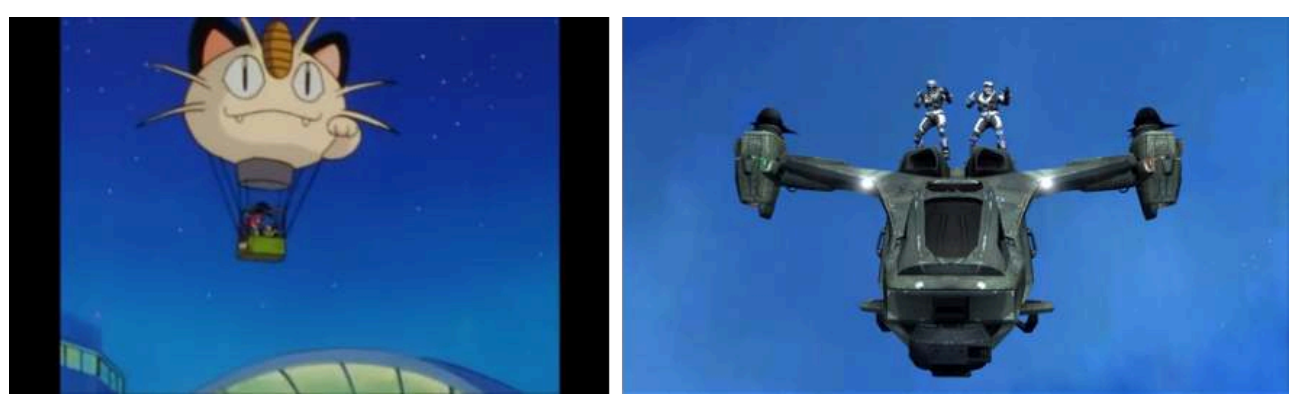

Fig. 6 (Tous Droits Réservés) - À gauche, la montgolfière utilisée par la Team Rocket pour se déplacer ; à droite, le Falcon sur lequel se tient la Team Croquette

Dans le même ordre d'idées, lorsque, dans le premier épisode, les "Gros-lâches » attaquent les héros en leur tirant dessus avec leurs armes, cette action n'a plus seulement le sens d'un frag ludique (il ne s'agit plus d'abattre un adversaire qui réapparaîtra peu de temps après pour reprendre la partie) : elle est thématisée comme une véritable tentative de meurtre. Les composantes du jeu ne fonctionnent donc pas systématiquement comme des métalepses: elles ne servent pas toujours à figurer l'expérience ludique dans la vidéo ou même à produire des effets de «sortie » de la fiction, mais participent à construire cette dernière.

Par exemple, le fait que le Professeur Chen soit incarné, dans Garrymon, par un personnage de Half-Life 2 (Kleiner) n'empêche pas celui-ci de constituer une figure autonome : les deux instances du personnage restent distinctes (le Kleiner de la vidéo a 
peu en commun avec celui du jeu) et la diégèse de Half-Life n'est, au final, jamais vraiment mentionnée comme telle. Ce n'est pas tant Kleiner qui est extrait de sa fiction pour être importé dans Garrymon, que le personnage du machinima qui est narrativisé, thématisé en renvoyant vers un autre univers à la manière d'une mascotte, d'un indice. Ce faisant, les protagonistes ou objets de la vidéo condensent, par leur seule apparition, un effet d'écart, en ce qu'ils sont à la fois similaires et éloignés de leurs ersatz vidéoludiques.

Ce principe est d'ailleurs au fondement d'un des premiers traits d'humour de Garrymon, puisque le protagoniste s'exclame, au début du premier épisode (01:31) : «maintenant que j'ai dix ans, je peux enfin obtenir ma licence Garrymon! » (« now that I'm ten, I can finally get my Garrymon license!»). Or son apparence (fig. 7) et son rapport intertextuel avec son homonyme John Freeman rendent paradoxale cette affirmation, pourtant sensée dans l'univers de Garrymon (aucun personnage ne la remettra en doute) et dans Pokémon.

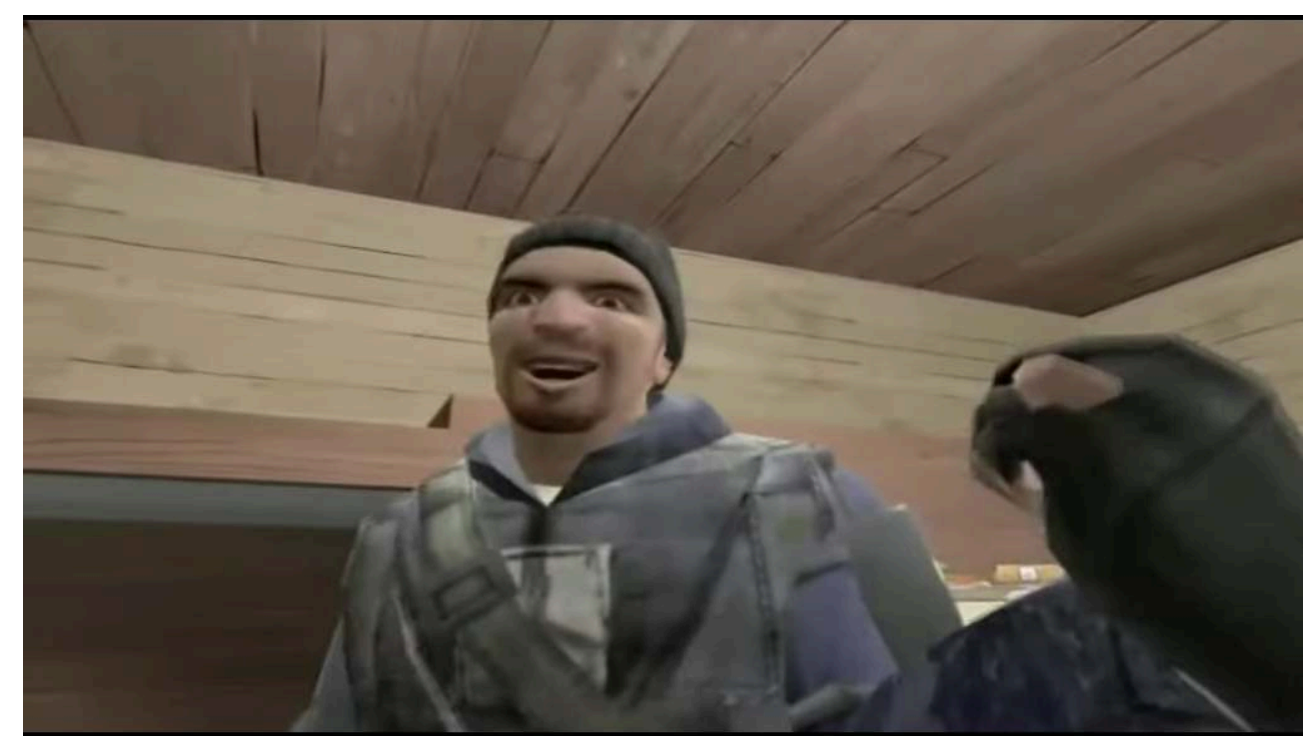

Fig. 7 (Tous Droits Réservés) - John Freeman vient d'avoir dix ans et peut donc demander sa licence Garrymon

En somme, pour reprendre la terminologie de Bardzell, les machinimas ne mobilisent pas les éléments du jeu sur un mode "objectif », c'est-à-dire en conservant leur utilisation et leur signification ludique (l'interface apparaîtrait bien comme une interface, les avatars resteraient des avatars, etc.). Ils les emploient plutôt sur un mode "subjectif»: ces éléments " font l'objet d'une appropriation, de telle sorte que leurs significations sont subverties, déformées ou par ailleurs altérées pour produire un sens qui n'est pas originaire de l'univers du jeu $»^{35}(2011: 206-207)$.

Malgré tout, de la même façon que la remédiatisation (Rajewsky 2005) ne fait pas pour autant disparaître les codes du jeu, les significations ludiques de ces composantes ne disparaissent pas pour être remplacées par celles que la vidéo leur attribue : elles restent lisibles à travers les nouveaux signes. Dans Halomon, Pikafion est à la fois une occurrence de Pikachu et un alien Sangheili de Halo ; dans Garrymon, Lamarr renvoie aussi à la fois à Pikachu et au headcrab de Half-Life (condensant les attributs monstrueux du second et mignons du premier : les autres personnages réagissent, en effet, comme s'il s'agissait d'une créature adorable). 
51 Le « découpage-collage » d'éléments du jeu dans le machinima et leur resémantisation construisent donc autant de «palimpsestes » narratifs, dont le fonctionnement n'est pas sans rappeler celui de la métaphore. Un signe (un modèle 3D) est utilisé pour renvoyer à un autre "sens dénotatif » que celui qui lui est couramment associé, et ce, en vertu d'un rapport de comparaison : l'alien Sangheili et le headcrab sont à entendre comme s'ils étaient des Pikachu, l'avatar Pyro de Team Fortress 2, modifié pour apparaître avec trois têtes, est comparé au Pokémon Dodrio (fig. 8), etc.
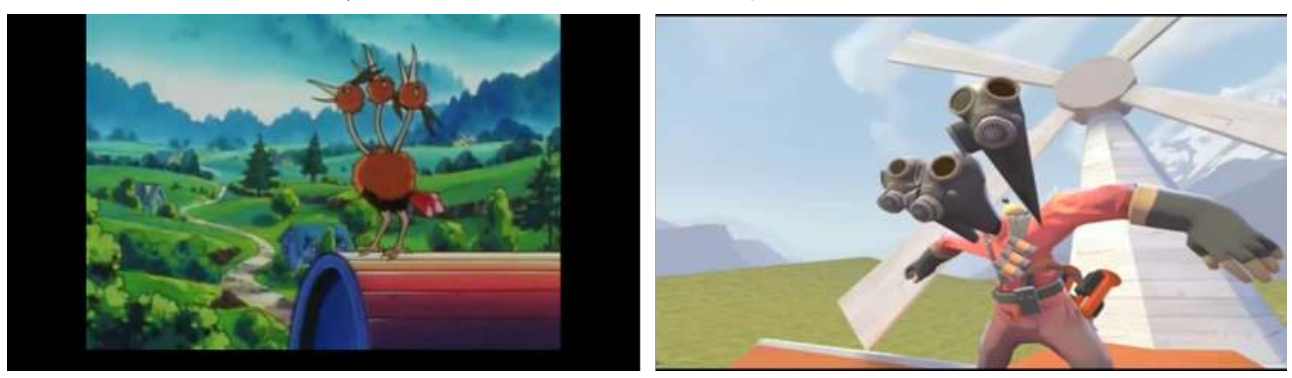

Fig. 8 (Tous Droits Réservés) - Le Pokémon Dodrio (à gauche) est représenté dans Garrymon (épisode 1, 02:27) par un Pyro à trois têtes (à droite)

52 Ce dernier exemple permet, en outre, de remarquer que les signes en question sont parfois modifiés afin d'augmenter le nombre de sèmes qu'ils partagent avec leurs comparés (c'est aussi le cas quand les doubleurs de Halomon imitent les voix des personnages de la série originale pour rendre les comparés de leurs avatars en armure plus identifiables). De telles transformations laissent toutefois des sutures apparentes, qui accentuent l'effet de collage produit par ces «métaphores intertextuelles». Une illustration parlante peut en être trouvée dans le deuxième épisode de Garrymon (09:55), lorsqu'un appel en visioconférence dévoile au protagoniste le Professeur Kleiner dénudé. Dans les faits, la tête du personnage est simplement collée sur une photo représentant un corps de pompier, dans un assemblage grossier et explicitement artificiel.

Selon Pinchbeck et Gras (2011 : 144), le mélange d'identités visuelles et sémantiques à l'œuvre dans la plupart des machinimas posséderait une dimension subversive intrinsèque, qui prédestinerait le genre à la satire et la comédie. Il est, en tout cas, notable que nombre de ces vidéos, loin d'atténuer les sutures de leurs greffes, les marques de leurs réécritures ou l'hétérogénéité de leurs emprunts, appuient au contraire celles-ci par des mécanismes de caricature.

\section{Caricatures et « éléments d'attraction »}

54 Les limitations du matériau expressif dont disposent les machinimas (le peu de gestes qu'il est possible de faire faire aux avatars, par exemple) nécessitent, bien souvent, le recours à l'exagération et à la simplification pour rendre l'action compréhensible. Pour cette raison, les situations ou personnages représentés dans ces vidéos sont généralement réduits à quelques éléments simples, mais distinctifs. Dans Halomon (et dans la plupart des machinimas réalisés avec Halo), les personnages sont principalement reconnaissables par la couleur de leur armure: la couleur bleue de l'agent Jenny rappelle son uniforme de police, l'armure blanche du Professeur À-la-chaîne fait référence à la blouse blanche du Professeur Chen, l'orange de la jeune fille rencontrée par les héros renvoie aux cheveux roux d'Ondine, etc. Les Pokémon, étant également 
représentés par des avatars de Halo, donc des êtres humanoïdes (humains ou aliens), ne sont aussi distingués que par leurs couleurs et leurs voix (fig. 9) : Pikafion est jaune comme Pikachu, les Leveinard qui apparaissent dans le deuxième épisode (appelés, pour l'occasion, «Levétard »; 05:50) sont simplement en armure rose pâle, etc.
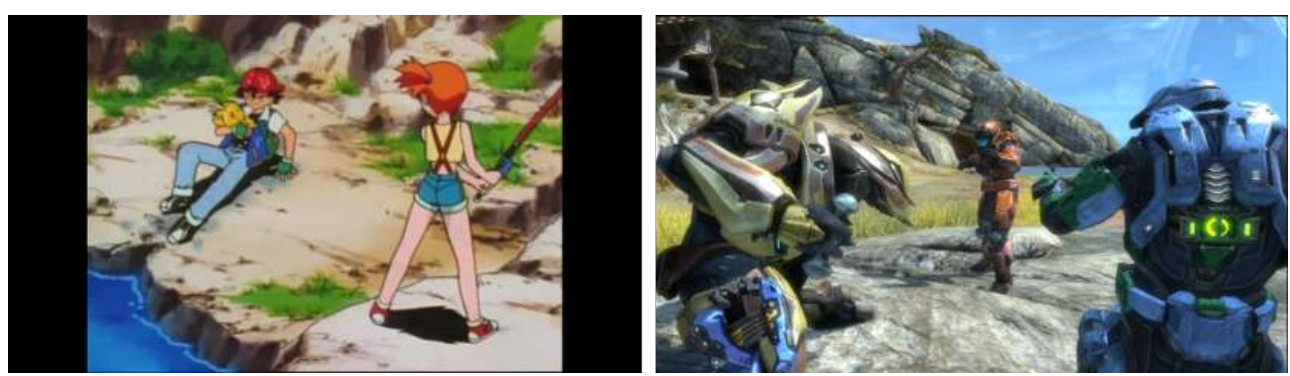

Fig. 9 (Tous Droits Réservés) - Sacha, Ondine et Pikachu sont réduits, dans le machinima, à leurs couleurs jugées dominantes : le bleu, l'orange et le jaune

Pour être lisible, en d'autres termes, la narration du machinima semble presque nécessairement devoir passer par la caricature - un peu à la manière du théâtre d'objet qui, pour pallier l'inexpressivité et la rigidité des marionnettes, doit exagérer leurs mouvements et leurs traits. La dimension caricaturale est particulièrement perceptible dans la manière dont le machinima représente les déplacements. Ceux-ci sont non seulement resémantisés, mais aussi lourdement appuyés: simuler un dialogue dans Halo demande de faire bouger le corps de tout l'avatar et, si le ragdoll posing de Garry's Mod permet de figurer des expressions faciales plus variées, le fait que celles-ci soient capturées en stop motion rend les changements d'humeur des personnages très saccadés et mécaniques (leur bouche s'ouvre et se ferme sur un mode binaire, notamment).

Usant de cette possibilité, les machinéastes n'hésitent pas, en outre, à modeler les visages de leurs personnages pour forcer leurs traits jusqu'à les rendre monstrueux : en témoignent le large sourire de John Freeman dès le début du premier épisode (01:25; fig. 10), son visage étiré lorsqu'il se réveille (02:45), l'expression difforme de sa compagne lorsqu'elle lui indique le Centre Garrymon (08:31) ou encore celle du Professeur Kleiner lorsqu'il lui confie Lamarr (04:51 ; fig. 10). Dans Garrymon encore, les autres types de déplacements sont soumis au même type de traitement: qu'ils volent pour marcher (épisode $1: 02: 13)$, tombent pour descendre une pente (06:08-06:17), gesticulent dans tous les sens pour montrer leur joie (03:49) ou se roulent par terre pour signaler leur douleur (06:47), les personnages manifestent leurs actions par des gestes amples et reconnaissables.
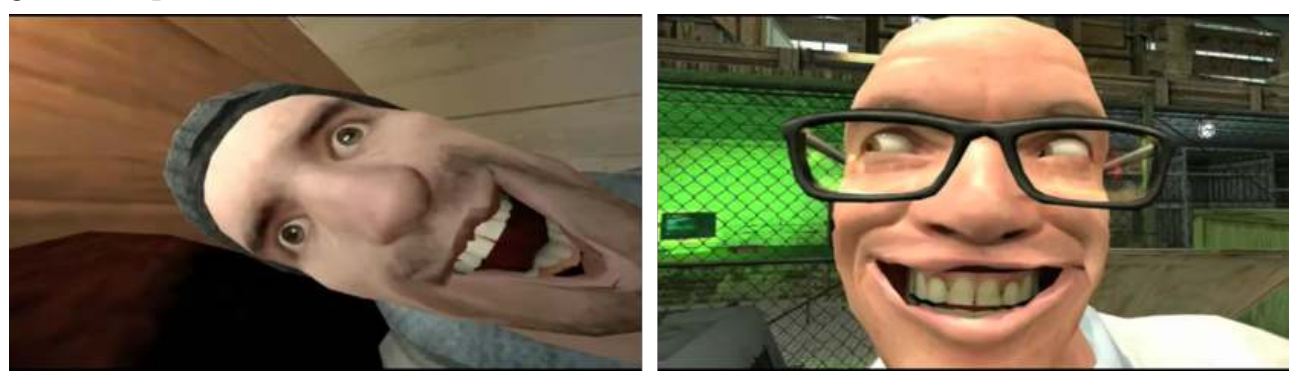

Fig. 10 (Tous Droits Réservés) - Les manipulations permises par le face posing sont délicates et peuvent rapidement déboucher sur des expressions difformes, mais cette difformité est cultivée par les machinéastes de Garry's Mod

Dans le contexte de la narration, la décomposition des personnages et situations en éléments prototypiques et significatifs n'est pas sans rappeler la notion d'« éléments 
d'attraction » développée par Azuma. Celui-ci remarque, en effet, que les personnages de la culture Otaku sont formés par l'agglomération de composantes préexistantes que le public a déjà appris à aimer par un effet de répétition (dans le cas des mangas, un personnage féminin peut ainsi se définir par la combinaison d'oreilles de chat, d'un costume de soubrette et de cheveux en forme d'antennes; Azuma 2008: 74-75). «Ces signes qui se sont développés pour stimuler habilement la fascination des consommateurs envers les personnages, je les désignerai [...] par le terme générique $\mathrm{d}^{\text {'“ }}$ éléments d'attraction" » (Azuma 2008 : 76).

Le concept semble s'appliquer aisément aux machinimas, qui condensent bien souvent de manière syncrétique des caractéristiques populaires des jeux. Il n'est pas innocent, par exemple, que la créature choisie pour représenter Pikachu dans Garrymon soit le headcrab Lamarr, car il s'agit d'un monstre emblématique de la série Half-Life, pour laquelle il joue fréquemment le rôle de mascotte. De même, il n'y a rien d'étonnant à ce que les deux véhicules qui apparaissent dans Halomon soient le Warthog et le Falcon, car ceux-ci sont aussi des composantes prototypiques du jeu Halo.

\section{Conclusion}

Par ses principes créatifs, le machinima est un exemple d'écriture polyphonique: différents médias, genres, intertextes y entrent en concurrence, mais aussi diverses logiques (celles des univers représentés, celles du jeu, du monde empirique ou encore de la doxa) et différents régimes de contraintes. De ces conflits énonciatifs naissent des vidéos à l'aspect bricolé, pleines d'aspérités et décalées par rapport à tous les modes d'expressions auxquels elles empruntent.

À travers l'analyse conjointe des séries Halomon et Garrymon, nous avons tenté de montrer que, lorsque ces œuvres tentent de construire un récit, leur polyphonie structurelle entraîne la production d'un type très particulier de diégèse. Ces vidéos sont saturées de figures qui ne cessent de mettre en doute l'existence d'un cadre pour la fiction (qui en garantirait l'unité de sens) ou qui, en renvoyant de façon indexicale à la matrice vidéoludique leur servant d'origine, manifestent que ce genre ne peut s'exprimer que par l'à peu près. Dans sa dimension de pastiche, le machinima met en jeu les médias qu'il imite : il fait comme s'il était une série télévisée, comme s'il racontait l'histoire de Pokémon, et son incapacité à totalement reproduire ses inspirations met clairement au jour l'« écart intermédial» (Rajewsky 2005: 55) qui ne cesse de l'en séparer.

61 De la combinaison de ces figures naît une fiction mixte (mêlant les univers de Pokémon, Halo et Garry's Mod, le monde empirique, les références communautaires des joueurs, la culture internet, etc.), hétérogène et peu cohérente sur le plan sémantique, mais qui trouve une logique propre dans l'effet de décalage et de satire qu'elle produit. Cette fiction tient de ce qu'Amato nomme une « ludiégèse » $(2005: 301)$ : une diégèse ludique qui répond à d'autres règles que les seuls codes narratifs, et où la blague, le bon mot ou l'introduction d'absurde priment bien souvent sur les impératifs de logique et de cohérence. Cette fiction, enfin, suppose une réception qui est elle-même qualifiable de ludique: l'univers représenté ne prend sens que si le récepteur accepte de «jouer le jeu » de la parodie, d'accepter les constants changements de paradigmes ou d'univers de référence opérés par les personnages et d'actualiser le comique intertextuel de 
l'œuvre en la comparant mentalement à toutes ses sources, à tous les substrats médiatiques qui la composent.

\section{BIBLIOGRAPHIE}

Amato, Étienne Armand (2005), « Reformulation du corps humain par le jeu vidéo : la posture vidéoludique ", in Le game design de jeux vidéo. Approches de l'expression vidéoludique, S. Genvo (dir.), Paris, L'Harmattan, p. 299-323

Azuma, Hiroki (2008), Génération Otaku. Les enfants de la postmodernité, Paris, Hachette Littératures Bardzell, Jeffrey (2011), « Machinimatic Realism : Capturing and Presenting the "Real World" of Video Games », in The Machinima Reader, H. Lowood \& M. Nitsche (dirs.), Cambridge, MIT Press, p. $195-217$

Barnabé, Fanny (2017), Rhétorique du détournement vidéoludique. Le cas de Pokémon, Thèse de doctorat en Langues et lettres, en ligne, consulté le 09 avril 2020, URL : http://hdl.handle.net/ $2268 / 210764$

Bateson, Gregory (1977), « Une théorie du jeu et du fantasme », in Vers une écologie de l'esprit, tome 1, Paris, Seuil, pp. 209-224, en ligne, consulté le 09 avril 2020, URL : http://www.ultramuros.ca/ documents/Bateson2-Theo-de-la-communication.pdf Bonhomme, Marc (2014 [2005]), Pragmatique des figures du discours, Paris, Honoré Champion Cameron, David \& Carroll, John (2009), « Encoding liveness : Performance and real-time rendering in machinima ", Proceedings of the 2009 DiGRA International Conference : Breaking New Ground: Innovation in Games, Play, Practice and Theory, en ligne, consulté le 09 avril 2020, URL : http://www.digra.org/wp-content/uploads/digital-library/09291.37018.pdf Compagnon, Antoine (1979), La seconde main ou le travail de la citation, Paris, Seuil De Certeau, Michel (1980), L'invention du quotidien. 1, Arts de faire, Paris, Union générale d'éditions U.G.E.

Derecho, Abigail (2006), « Archontic literature. A definition, a history, and several theories of fan fiction ", in Fan Fiction and Fan Communities in the Age of the Internet, K. Hellekson \& K. Busse (dirs.), Jefferson, McFarland, p. 61-78

Eco, Umberto (1985 [1979]), Lector in fabula : le rôle du lecteur ou la Coopération interprétative dans les textes, trad. M. Bouzaher, Paris, Librairie Générale Française Fevry, Sébastien (2007), La mise en abyme filmique. Essai de typologie, Liège, CEFAL Genette, Gérard (1982), Palimpsestes. La littérature au second degré, Paris, Seuil Genette, Gérard (2004), Métalepse : de la figure à la fiction, Paris, Seuil Genvo, Sébastien (2011), « Penser les phénomènes de "ludicisation" du numérique : pour une théorie de la jouabilité ", Revue des sciences sociales, $n^{\circ}$ 45, pp. 68-77, en ligne, consulté le 09 avril 2020, URL : http://www.ludologique.com/publis/Ludicisation_Genvo_S.pdf 
Georges, Fanny \& Auray, Nicolas (2012a), « Approche sémiopragmatique de l'espace de communication des machinima ", Revue des Interactions Humaines Médiatisées, n¹3(1), pp. 03- en ligne, consulté le 09 avril 2020, URL : http://europia.org/RIHM/V13N1/RIHM13\%281\%292Georges.pdf

Georges, Fanny \& Auray, Nicolas (2012b), « Les productions audiovisuelles des joueurs de jeux vidéo. Entre formation professionnelle et apprentissages esthétiques autodidactes ", Réseaux, $\mathrm{n}^{\circ} 175$, pp. 145-173, en ligne, consulté le 09 avril 2020, URL : http://www.cairn.info/revuereseaux-2012-5-page-145.htm\#re1no1

Kelland, Matt (2011), « From Game Mod to Low-Budget Film : The Evolution of Machinima », in The Machinima Reader, H. Lowood \& M. Nitsche (dirs.), Cambridge, MIT Press, p. 23-35

Kelland, Matt, Dave Morris \& Dave Lloyd (2005), Machinima, Boston, Thomson

Kirschner, Friedrich (2011), « Toward a Machinima Studio », in The Machinima Reader, H. Lowood \& M. Nitsche (dirs.), Cambridge, MIT Press, p. 53-71

Krapp, Peter (2011), " Of Games and Gestures : Machinima and the Suspension of Animation », in The Machinima Reader, H. Lowood \& M. Nitsche (dirs.), Cambridge, MIT Press, p. 159-174

Lowood, Henry (2006), « High-performance play. The making of machinima ", Journal of Media Practice, $\mathrm{n}^{\circ}$ 7(1), pp. 25-42, en ligne, consulté le 09 avril 2020, URL : http://www.tandfonline.com/ doi/pdf/10.1386/jmpr.7.1.25/1

Lowood, Henry (2008), « Found Technology. Players as Innovators in the Making of Machinima », in Digital Youth, Innovation, and the Unexpected, T. McPherson (dir.), Cambridge, MIT Press, p. 165-196, en ligne, consulté le 09 avril 2020, URL : https://www.academia.edu/930089/ Found_technology_Players_as_Innovators_in_the_making_of_machinima

Lowood, Henry (2011), « Video Capture : Machinima, Documentation, and the History of Virtual Worlds ", in The Machinima Reader, H. Lowood \& M. Nitsche (dirs.), Cambridge, MIT Press, p. 03-22 Manovich, Lev (2011), « Image Future », in The Machinima Reader, H. Lowood \& M. Nitsche (dirs.), Cambridge, MIT Press, p. 73-90

Marino, Paul (2004), 3D Game-Based Filmmaking : The Art of Machinima, Scottsdale, Paraglyph Press Mazalek, Ali (2011), « Tangible Narratives : Emerging Interfaces for Digital Storytelling and Machinima », in The Machinima Reader, H. Lowood \& M. Nitsche (dirs.), Cambridge, MIT Press, p. $91-110$

Newman, James (2008), Playing with videogames, Londres, Routledge

Nitsche, Michael (2005), « Film live : And Excursion into Machinima », in Developing Interactive Narrative Content : sagas_sagasnet_reader, B. Bushoff (dir.), Munich, HighText, pp. 210-243, en ligne, consulté le 09 avril 2020, URL : http://homes.lmc.gatech.edu/ nitsche/download/ Nitsche_machinima_DRAFT4.pdf

Nitsche, Michael (2007), « Claiming Its Space: Machinima », Dichtung Digital, $n^{\circ}$ 37, en ligne, consulté le 09 avril 2020, URL : http://www.dichtung-digital.org/2007/Nitsche/nitsche.htm Nitsche, Michael (2011), « Machinima as Media », in The Machinima Reader, H. Lowood \& M. Nitsche (dirs.), Cambridge, MIT Press, p. 113-125

Picard, Martin (2007), « Machinima: Video Game As An Art Form ? », Loading..., n 1(1), en ligne, consulté le 09 avril 2020, URL : http://journals.sfu.ca/loading/index.php/loading/article/view/ $17 / 20$ 
Pigott, Michael (2011), « How Do You Solve a Problem Like Machinima?», in The Machinima Reader, H. Lowood \& M. Nitsche (dirs.), Cambridge, MIT Press, p. 177-193

Pinchbeck, Dan \& Ricard Gras (2011), « Machinima : From Art Object to Cultural Practice », in The Machinima Reader, H. Lowood \& M. Nitsche (dirs.), Cambridge, MIT Press, p. 143-158

Rajewsky, Irina O. (2005), « Intermediality, Intertextuality, and Remediation : A Literary Perspective on Intermediality ", Intermédialités : histoire et théorie des arts, des lettres et des techniques/Intermediality : History and Theory of the Arts, Literature and Technologies, $\mathrm{n}^{\circ}$ 6, pp. 43-64, en ligne, consulté le 09 avril 2020, URL : http://id.erudit.org/iderudit/1005505ar

Saint-Amand, Denis (2013), Le dictionnaire détourné. Socio-logiques d'un genre au second degré, Rennes, Presses Universitaires de Rennes

Salen, Katie (2011), « Arrested Development : Why Machinima Can't (or Shouldn't) Grow Up », in The Machinima Reader, H. Lowood \& M. Nitsche (dirs.), Cambridge, MIT Press, p. 37-50

Schaeffer, Jean-Marie (1999), Pourquoi la fiction?, Paris, Seuil

Schneider, Erik (2008), Story and Game Combined. Using Machinima for Interactive Experiences, Mémoire de Master en Arts, Hochschule der Medien Stuttgart, en ligne, consulté le 09 avril 2020, URL : http://fba.unlp.edu.ar/lenguajemm/?wpfb_dl=58

Schott, Gareth (2011), « The Production of Machinima : A Dialogue between Ethnography, Culture and Space », International Journal of Business, Humanities and Technology, $n^{\circ} 1(1)$, p. 113-121

Schott, Gareth \& Bevin Yeatman (2011), « Participatory Fan Culture and Half-Life 2 Machinima : A Dialogue among Ethnography, Culture and Space », in The Machinima Reader, H. Lowood \&

M. Nitsche (dirs.), Cambridge, MIT Press, p. 301-314

\section{NOTES}

1. Pour plus de détails sur les différentes formes de détournement de jeux vidéo, nous renvoyons à notre thèse de doctorat (Barnabé, 2017).

2. L'expression est celle de Genette (1982: 13) et désigne "une production littéraire dérivée d'une autre, tout en laissant entendre le potentiel satirique (plus ou moins effectif selon les cas [...]) de ces textes seconds» (Saint-Amand, $2013: 48$ ).

3. La vidéo « Kill Phil » du studio TGO GMBH, par exemple, reproduit dans Halo la mise en scène $\mathrm{du}$ film Kill Bill (Quentin Tarantino, 2003). Consulté le 09 avril 2020, URL: https:// www.youtube.com/watch?v=Q_aZENTuBZs.

4. À l'image du long métrage " †CARRIE† », qui réécrit le roman éponyme de Stephen King (1974) dans Les Sims 2. Consulté le 09 avril 2020, URL: https://www.youtube.com/watch? $\mathrm{v}=\_u U 9 b z L_{-}$pH0

$\& \mathrm{t}=2185 \mathrm{~s}$.

5. «Halomon (Ep 1) - Machinima Halo Reach », consulté le 09 avril 2020, URL: https:// www.youtube.com/watch?v=sRoPYTi3Z7U et "Halomon (Ep 2) - Machinima Halo Reach», consulté le 09 avril 2020, URL : https://www.youtube.com/watch?v=T0B6vWWNcoc.

6. «Garrymon Pilot Episode », consulté le 09 avril 2020, URL : https://www.youtube.com/watch? v=Edym6_vnjUw et «Garrymon Episode 2 (Garry's Mod Machinima) », consulté le 09 avril 2020, URL : https://www.youtu be.com/watch?v=NVx5WKPaF8w\&t=911s.

7. Acronyme de « First Person Shooter » (« jeu de tir à la première personne »). 
8. Notons que cette réécriture du patronyme Chen renvoie à la traduction française d'overkill, nom d'une des médailles de Halo: Reach, que le joueur peut débloquer à condition de tuer quatre ennemis avec moins de quatre secondes d'intervalle entre chaque élimination.

9. Par souci d'alléger le texte, nous mentionnerons systématiquement les timecodes des passages commentés dans ce format (minutes:secondes).

10. Les composants de Half-Life 2 sont disponibles par défaut, mais il est également possible de télécharger des packs de ressources graphiques issues d'autres jeux.

11. Dans les jeux vidéo, le terme ragdoll renvoie aux corps des personnages lorsqu'ils ne sont plus animés, mais se comportent comme des «poupées de chiffon » soumises aux mouvements que leur impose la physique du jeu (la gravité, etc.). Dans certains titres, les personnages deviennent des ragdolls lorsqu'ils meurent et peuvent être manipulés ou déplacés par le joueur.

12. Voir, pour exemple, ce fanart de stabkamay sur DeviantArt: "Gmod - Scout's Workout", consulté le 09 avril 2020, URL: http://stabkamay.deviantart.com/art/Gmod-Scout-sWorkout-382168615.

13. Pour illustration, voir la bande dessinée Concerned sur Screen Cuisine : «Concerned \#6: Sunday, May 8, 2005 », consulté le 09 avril 2020, URL : http://www.screencuisine.net/hlcomic/index.php? date $=2005-05-08$.

14. La série de machinimas The GMod Idiot Box du machinéaste DasBoSchitt en constitue un bon exemple. Consulté le 09 avril 2020, URL : https://www.youtube.com/watch?v=8rS1UYYiytw.

15. Consulté le 09 avril 2020, URL : https://www.youtube.com/watch?v=OHxyZaZlaOs.

16. Un crossover est une œuvre qui mélange plusieurs univers fictionnels.

17. Le fait que ces vidéos soient contraintes par le gameplay et le moteur physique du jeu utilisé.

18. "Machinima is the process of rendering a linear artifact from a nonlinear system, of reducing the field of affordances within this system to an individualized stream ». Signalons que toutes les traductions de citations anglaises présentes dans cet article sont de notre fait.

19. "The multiplicity of choices potentially generated through interaction within a virtual environment become contained as a singular and repeatable trajectory of actual choices made by the producer of machinima ".

20. Selon Genvo, en effet, l'existence d'indétermination conditionne la jouabilité d'une situation : "puisque jouer c'est faire (Winnicott, 1971), il est selon nous nécessaire d'ajouter que jouer c'est aussi prendre une décision et "faire l'exercice du possible". Si le jeu ne consiste que dans la succession de décisions uniques, alors le joueur n'a aucune "latitude" dans ses choix; il se contente d'actualiser une proposition tenue pour vraie, qui ne dépend pas de son jeu particulier » (Genvo, 2011: 72).

21. «In fan fiction, there is an acknowledgment that every text contains infinite potentialities, any of which could be actualized by any writer interested in doing the job : fic authors [...] explore situations that the makers of the source text simply cannot, because of the need for continuity and chronological coherence in the source text's universe (and the lack of such a requirement in fan productions) ».

22. Voire : d'une ouverture et d'une multiplication des «mondes possibles» dont elle est la matrice, pour reprendre les termes d'Eco (1985:226).

23. TomTom est une marque de systèmes de navigation GPS.

24. Le nom Lambda fait référence au logo de Half-Life $(\lambda)$, qu'on retrouve à de nombreuses occasions dans les jeux, notamment sur la combinaison de l'avatar, Gordon Freeman.

25. "It's got a guitar on the side to make it look cool, a super-deluxe hand designed specifically to catch criminals, a heavily modified TomTom to detect criminal behavior within five hundred yards, and a fifteen-megawatt thruster on the back to make you fly through the Lambda region really fast ".

26. «I wanna be the very best, like Half-Life never was ». 
27. Dans un jeu vidéo, le terme spawn est utilisé (et francisé) pour désigner l'apparition d'un objet ou d'un personnage.

28. Codes de triche intégrés dans les jeux produits sur Source.

29. Système d'écriture qui consiste à utiliser, à la place des lettres, des chiffres ou caractères spéciaux qui leur ressemblent graphiquement : leet peut ainsi s'écrire « 1337 ».

30. «To prevent all users from overspawning, / To make sure games are never boring, / To denounce the evils of sv_cheats, / To extend our knowledge of speaking leet, / Judith ! / Breen! / Team Thruster thrusting at the speed of light, / Surrender now or prepare to fight, / Meow, that's eh... hem... correct... ».

31. «A key factor in most machinima production is that the game environment is not a passive performance space; the game engine may push back at the actions of the performer/player in ways that hinder the machinima production, but which would be consistent with game oriented behaviour ".

32. La ludicisation est définie par Genvo comme l'ensemble des «processus qui consistent à faire entrer un objet dans le monde du jeu, en soulignant que la définition de cette notion et des objets auxquels elle renvoie est hautement contextuelle et peut être amenée à évoluer » (Genvo, 2011 : 62).

33. Dans le clip musical «Spartan Style» de la TeamHeadKick, certains bugs graphiques sont utilisés pour représenter des mouvements de « danse » chez les avatars. Consulté le 09 avril 2020, URL : https://www.youtube.com/watch?v=CuVd9oK16V0. Des gestes ludiques peuvent aussi servir de prétexte à toute une mise en scène venant leur donner du sens : c'est la physique particulière du véhicule Warthog dans Halo qui a inspiré la célèbre vidéo "Warthog Jump " (consulté le 09 avril 2020, URL : https://www.youtube.com/

watch?v=nGQIQljaAc0).

34. "Where the player-controlled character might be thought of algorithmically in terms of gameplay-affecting capacities, capabilities or properties during play or superplay [...], in Machinima, they are reframed as part of a representational system $»$.

35. «[...] these assets are subject to appropriation, such that their meanings are subverted, distorted, or otherwise altered to produce a meaning that is not native to the game world ».

36. Mode qui n'est pas sans rappeler celui de l'attitude ludique : le jeu peut effectivement être envisagé comme un mode d'interaction au second degré où les actions réalisées et les objets manipulés ne sont plus des référents, mais des signes d'eux-mêmes (voir Bateson, 1977 : 211 ; Genvo, 2011 : 70 ou encore Schaeffer, 1999 : 145-147).

\section{RÉSUMÉS}

À travers l'analyse de deux séries de machinimas (Halomon et Garrymon) ayant pour point commun de remédiatiser la série animée Pokémon dans d'autres jeux vidéo, le présent travail vise à étudier l'articulation des procédés rhétoriques et narratifs de cette forme de détournement vidéoludique. Précisément, l'article s'interroge sur la manière dont la polyphonie intrinsèque du machinima (qui renvoie toujours simultanément à plusieurs substrats médiatiques et à plusieurs univers fictionnels) détermine sa narration et caractérise par avance le type de fiction que ce format peut construire. Différentes figures rhétoriques faisant «saillance" au sein des vidéos sont ainsi identifiées afin de montrer leur impact sur la construction du récit. Ces procédés sont réunis en deux groupes : les figures opérant des ruptures du cadre narratif (fragmentations, 
paradoxes, métalepses, et mises en abyme) et celles manifestant l'indexicalité du machinima, sa dimension de « narration sous contrainte » (resémantisations, métaphores et caricatures).

Through the analysis of two series of machinimas (Halomon and Garrymon) remediating the animated series Pokémon in other video games, this research aims to study the articulation of the rhetorical and narrative processes of this form of video game remix. Specifically, this paper questions how the intrinsic polyphony of machinima (which always refers simultaneously to several media substrates and fictional universes) determines its narrative and characterizes in advance the type of fiction that it can construct. Various rhetorical figures that are "salient" within the videos are identified in order to show their impact on the construction of the narrative. These processes are grouped into two categories: figures producing ruptures in the narrative framework (fragmentation, paradoxes, metalepses, and mises en abyme) and figures manifesting the indexicality of machinima, underlining the "narration under constraint" aspect of such productions (resemantizations, metaphors and caricatures).

INDEX

Mots-clés : machinima, narration, pastiche, parodie, détournement, remédiatisation

\section{AUTEUR}

\section{FANNY BARNABÉ}

Fanny Barnabé est Chargée de recherches FNRS à l'Université de Liège et membre du Liège Game Lab. Ses recherches portent sur la narration vidéoludique (à laquelle elle a consacré l'ouvrage Narration et jeu vidéo : Pour une exploration des univers fictionnels, paru aux Presses Universitaires de Liège), sur les différentes formes de détournement du jeu vidéo (sujet de sa thèse de doctorat) et sur les tutoriels de jeu vidéo. 